chain structure with little detergent bound, or more likely a two-chain structure plus a large amount of bound NP-40.

If the antigen-detergent complex does not contain a four-chain structure then there is the difficulty of explaining the reason for the appearance of the 45,000 chain in SDS polyacrylamide gel electrophoresis. Snary et al. have suggested that this may be due to disulphide exchange either in stored membrane, or possibly during immunoprecipitation procedures. Clearly the experiments must be repeated in the presence of oxidising agents.

If one accepts that histocompati- bility antigens may have a two-chain rather than a four-chain structure does this argue against a relationship between the antigens and immunoglobulin? Obviously not, and this merely points up the circumstantial nature of the current evidence. The matter can only be settled by amino acid sequence analysis of the 45,000 molecular weight glycoprotein which carries the antigens, and comparison of this with immunoglobulin sequences. Given the current progress in the purification of HLA and H-2, and also in microsequencing techniques, such data should be forthcoming in the next few years.

\title{
Chemical language in insects
}

from our Insect Physiology Correspondent

Iт has long been known that scents produced by glandular organs play an important part in animal communication. The naming of such chemicals, used for communication between members of the same species, as "pheromones' by Karlson and Lüscher coincided with the development of gas liquid chromatography, mass spectrometry and other methods which have made possible the chemical analysis of minimal quantities of material. The result has been a very rapid expansion of knowledge in this sphere. Whereas it took Butenandt and his associates many years and vast amounts of material to identify the chemical structure of 'bombycol', the female sex attractant of the silkworm moth, by classical procedures, complex mixtures can now be analysed in a matter of days.

The chemicals present in the glandular secretions of insects are often exceedingly diverse; 20-30 recognised components in a single gland. They comprise a vast range of saturated and unsaturated hydrocarbons and their derived alcohols and aldehydes (ranging from hexenal and tridecene to pentadecane), fatty acids (such as butyric and caproic acids), a great number of phenolic substances and quinones (such as phenol, $m$-cresol, benzoquinone and toluquinone) and a variety of terpenoids (such as geraniol, farnesol, citral, and citronellol).

So far as the perceptions of man are concerned, in recognising the odours characteristic of particular kinds of shops, or appreciating the bouquet of a vintage wine, it seems to be the complex mixture which is assessed. Insects seem often to be concerned with a single component: in the mandibular gland secretion of the queen honey bee (as shown by Butler and his associates) there are more than 30 compounds; of these, 9-ketodecenoic acid is the 'queen substance' which attracts males during the nuptial flight and inhibits royal-cell building by workers in the nest, whereas 9-hydroxydecenoic acid causes clustering and stabilisation of worker swarms.

The action of pheromones is commonly divided into two classes (following Wilson and Bossert): chemical 'releasers' of specific acts of behaviour, and 'primers' which seem to act initially on the endocrine system. The same pheromone may show both types of activity - as in the case of the queen substance which, besides its effects on behaviour, inhibits ovarian development in the workers. Moreover the same chemical can be used both as a pheromone for communication within the species and as an 'allomone' (as defined by Brown and Eisner) to provide defence against an enemy. That combination is well seen in the use of formic acid by the wood ant. Thus the effects of a pheromone depend on circumstances, on differences in concentration, and in some instances on the combination of secretions from different glands. It has even been suggested by Bossert that concentrations might be modulated in time. It is unlikely that the modulation of odour emission could attain the level of a symbolical or syntactical language but as $\mathrm{E}$. $\mathrm{O}$. Wilson points out the acoustical system has acquired quite complex signal modulation in the songs of insects.

The signal provided by a pheromone is the 'active space' as defined by Bossert and Wilson: the zone around the point of emission within which the concentration of pheromone molecules is above the threshold level of response. This zone may vary in shape with movement of the air, and in composition denending on the comparative rates of diffusion of the varied molecules. As Wilson has pointed out "the relation of chemosensory physiology to ecology can be fully elucidated only through the analysis of the active spaces".

These ideas have been carried some steps further by interesting observations of Bradshaw, Baker and Howse on the multicomponent pheromones of the weaver ant Oecophylla, as described on page 230 of this issue of Nature. These authors find evidence of caste differences in the mandibular gland secretions as well as differences in the components to which the castes respond. Furthermore, they have made interesting advances in studying the changes in the 'active spaces' of particular components which appear to provide sequential messages determined by the volatility of materials and the threshold concentrations for different responses. Worked out in detail in relation to the mode of life of a polymorphic species, all this shows the immense potentialities of chemical communication in the regulation of behaviour in a social insect.

\section{Fibroblast model for hormone effects}

\section{from Robert Shields}

ONE of the most striking features of the action of trophic hormones on cells is that many of the resulting changes in metabolism are remarkably similar, regardless of the type of hormone or the target tissue. Thus changes produced by oestrogen in the uterus, corticosteroids in the liver and insulin in muscle are much the same. These changes include alterations in macromolecular synthesis and a great deal of endocrinological research has been directed at finding out how hormones regulate these processes. The classical endocrinological approach has been to remove the gland producing the hormone under study and after a suitable period re-add the hormone and follow the subsequent changes in the target tissue. Although much qualitative data has been gathered in this way, the lack of suitable in vitro systems has hampered a quantitative assessment of the inter-relationship between the hormone-induced changes in RNA, protein and DNA synthesis.

One well known in vitro system that has been studied intensively (but for rather different reasons) is the control of growth of fibroblasts in tissue culture. It now seems apparent that the growth of these cells is regulated by the levels of serum factors available to the cell (News and Views, 